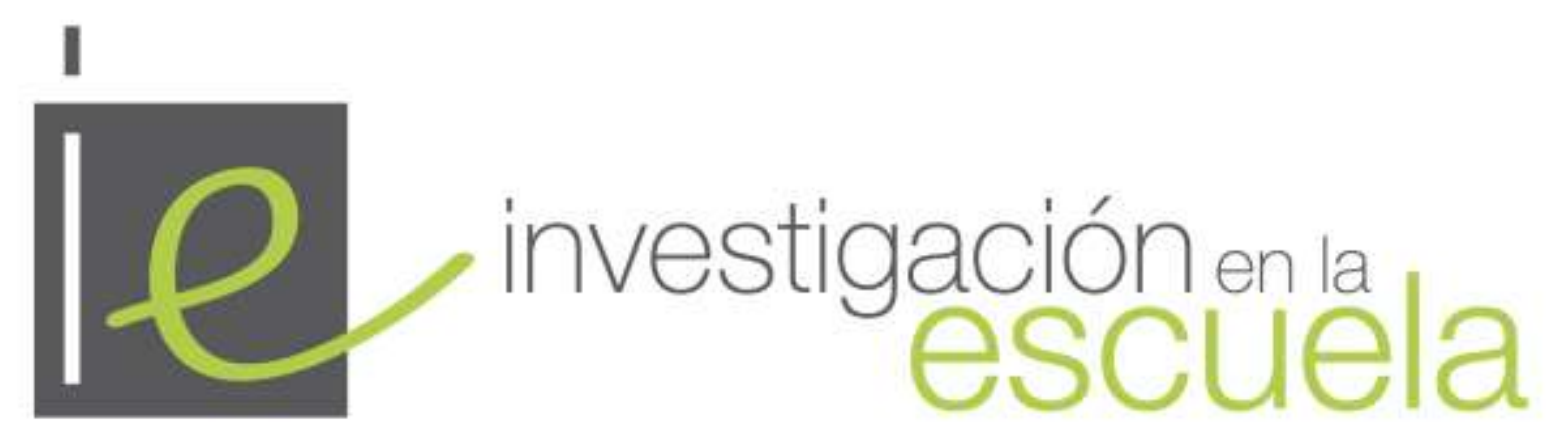

Revista internacional de investigación e innovación educativa

\title{
Entrenamiento de los movimientos oculares para el aumento de la velocidad lectora en Educación Primaria
}

\author{
Ramón Cruz del Moraly Sandra María Arjona Luque \\ Consejería de Educación de la Junta de Andalucía \\ España
}

Citación: Cruz del Moral, R. y Arjona-Luque, S.M. (2018). Entrenamiento de los movimientos oculares para el aumento de la velocidad lectora en Educación Primaria. Investigación en la Escuela, 96, $50-66$.

Resumen: Se presenta un trabajo con una metodología basada en la Investigación Acción en el que se implementa un programa de entrenamiento para la mejora de los movimientos sacádicos. La finalidad del mismo es aumentar de manera significativa la velocidad lectora del alumnado de Educación Primaria que puntúa por debajo del intervalo medio en este ámbito. Se realizó un análisis de estadística descriptiva de las tres variables dependientes (palabras leídas por minuto, tiempo de lectura y errores cometidos), se desarrolló un estudio de Modelo Lineal General con medidas repetidas (septiembre, diciembre y marzo) y la eta al cuadrado parcial para determinar el tamaño del efecto que causa la intervención, utilizándose el test de King-Devick para realizar las mediciones. Se obtuvieron mejoras estadísticamente significativas $(\mathrm{p}<0,01)$ en los resultados de las tres variables al comparar las medidas realizadas en septiembre y diciembre, mientras que fueron muy significativas $(p<0,001)$ al hacerlo con los resultados de la primera y la tercera variable en los meses de septiembre y marzo. Por último, se comprueba que el tamaño del efecto producido por el programa es grande. Con la aplicación de este programa de entrenamiento se determina una mejora en todas las variables dependientes, favoreciendo la fluidez lectora que es imprescindible para la comprensión de textos. 
Palabras clave: "Velocidad de lectura"; "técnicas de lectura"; "lectura funcional"; "dificultad para la lectura"; "investigación sobre programas de estudios".

Training eye movements to increase reading speed in Primary Education

Abstract: This article presents a work with Research-Action methodology in which a training program for the improvement of saccades movements is designed The purpose of which is to significantly increase the reading speed of Primary Education students who score below the average range in this area. An analysis of descriptive statistics of the three dependent variables (words read per minute, reading time and errors made), a study of the General Linear Model with repeated measures (September, December and March) and the partial square eta to determine the size of the effect caused by the intervention, using the King-Devick test to apply the measurements. Statistically significant improvements were obtained $(\mathrm{p}<.01)$ in the results of the three variables when comparing the measurements made in September and December, while they are very significant ( $p$ $<.001)$ when comparing the results of the first and third variables in the months of September and March. Finally, it is verified that the size of the effect produced by the program is large. With the application of this training program an improvement in all the dependent variables is determined, favoring the reading fluency processes, essential for the comprehension of texts.

Key words: "Reading speed"; "reading strategy"; "functional reading"; "reading difficulty"; "curriculum research".

\section{Entraînement des mouvements oculaires pour augmenter la vitesse de lecture dans l'enseignement primaire}

Resumè: Un travail avec la méthodologie Action Research est présenté dans lequel un programme de formation pour l'amélioration des mouvements de saccades est mis en œuvre. Dont le but est d'augmenter significativement la vitesse de lecture des élèves de l'enseignement primaire qui obtiennent un score inférieur à l'intervalle moyen. cette zone. Une analyse des statistiques descriptives des trois variables dépendantes (mots lus par minute, temps de lecture et erreurs effectuées) a été réalisée, une étude du modèle linéaire général avec des mesures répétées (septembre, décembre et mars) et l'éqa carré partiel a été développée. pour déterminer la taille de l'effet causé par l'intervention, en utilisant le test King-Devick pour effectuer les mesures. Des améliorations statistiquement significatives ont été obtenues $(\mathrm{p}<.01)$ dans les résultats des trois variables lors de la comparaison des mesures effectuées en septembre et en décembre, alors qu'elles étaient très significatives $(\mathrm{p}<.001)$ avec les résultats du premier et du troisième variable au cours des mois de Septembre et Mars. Enfin, il est vérifié que l'ampleur de l'effet produit par le programme est important. Avec l'application de ce programme de formation, une amélioration de toutes les variables dépendantes est déterminée, favorisant la fluidité de la lecture, essentielle à la compréhension des textes.

Mots-clés: "Vitesse de lecture"; "stratégie de lecture"; "lecture fonctionnelle"; "difficulté de lecture"; "recherche sur les programmes d'études".

\section{Introducción}

La siguiente investigación consiste en el análisis de la velocidad lectora (VL) del alumnado de Educación Primaria (EP). La finalidad de la misma es comprobar la relación entre la VL y la comprensión lectora (CL). Para conseguirlo, se aplican las pautas del método científico en el contexto escolar (Melero, 2011). 
La incidencia que tiene la VL en la CL la podemos encontramos en estudios como en los de Pascual, Goikoextea, Corral, Ferrero y Pereda (2014), Pinnell, Pikulski, Wixson, Campbell, Gough y Beatty (1995) y en el National Reading Panel (2000) que muestran una correlación significativa entre el número de palabras leídas por minuto y la CL de los sujetos de investigación. Gómez (2008, p. 101) nos dice que la relación entre VL y CL se debe a que la decodificación y la comprensión utilizan la misma capacidad de procesamiento y el cerebro humano presenta una capacidad limitada para interiorizar información. Por lo tanto, siguiendo con Gómez, si la decodificación no es automática, se ocupa una parte importante de los procesos de trabajo, reduciendo las posibilidades de comprender y memorizar. Esta idea también la recoge Alonso (2005, p. 81): si la velocidad es escasa se reduce el tiempo para realizar las funciones necesarias que acceden el mensaje escrito.

Por otro lado, podemos afirmar que cuando el alumnado comprende lo que lee es más probable que desarrolle actitudes positivas hacia la lectura (Guzmán, Fajardo y Duque, 2015, p. 63). Por el contrario, si presenta dificultades en este ámbito puede presentar pobre motivación, quedando comprometido su desarrollo emocional y académico, tal y como demuestran Galindo y Martínez (2014, p. 23).

En cuanto a la evaluación de la lectura en EP, autores como Cortés (2002), defienden que la rapidez junto con la precisión en la lectura son aspectos fundamentales al evaluar la misma. Por tanto, el sustrato de una buena CL son la velocidad y la precisión.

Por lo expuesto, se diseña un programa de entrenamiento con los siguientes objetivos:

a) Aumentar de manera significativa la VL del alumnado que puntúa por debajo del intervalo medio en este ámbito.

b) Reducir de manera significativa el número de errores cometidos en las lecturas realizadas por nuestros sujetos.

\section{Marco teórico}

El programa de mejora (PM) que se presenta se centra en el entrenamiento de los movimientos sacádicos. Estos movimientos se definen como los desplazamientos oculares que realizamos durante la lectura, mientras que las paradas que se producen se denominan fijaciones (Palomo, 2010, p. 24). Diversos estudios han puesto de manifiesto la estrecha relación existente entre la VL y las fijaciones que realizan los ojos en el texto (Bernardo, 2004; Kulp y Schmith, 1997; La Cámara, 2016; Paulidis, 1981; Scheiman y Wick, 1994). Estas fijaciones adquieren importancia porque en el escaso tiempo que transcurre en cada una de ellas, nuestro cerebro adquiera la información necesaria para darle sentido a lo que está leyendo, mientras que ese proceso de atención se inhibe durante el salto entre ellas (Paulidis, 1981). Siguiendo estas afirmaciones, Kuperman, Van Dyke y Henry (2016) defienden que se dispondrá de un buen rendimiento lector si se realizan rápidos movimientos oculares. Por lo tanto, se hace necesario que la decodificación del texto se automatice para que el lector/a utilice todos sus recursos cognitivos en la comprensión (Pinnell et al., 1995).

Por otro lado, y con la intención de instaurar hábitos lectores adecuados entre nuestro alumnado, podemos afirmar que cuando un discente comprende lo que lee es más probable que desarrolle actitudes positivas hacia la lectura (Guzmán et al., 2015, p. 63). Por el contrario, si el sujeto presenta dificultades en este ámbito, puede presentar pobre motivación, quedando comprometido su desarrollo emocional y académico porque, tal y como demuestran Galindo y Martínez (2014, p. 23), existe correlación significativa entre la lectura y el rendimiento escolar.

Teniendo en cuenta que en la actualidad la cantidad de información que se genera con la lengua escrita es inmensa, se debe priorizar la mejora del nivel de desempeño competencial lector 
para que nuestros alumnos/as puedan analizarla desde una perspectiva crítica (León, 2004). Para conseguirlo, se implementarán estrategias que mejoren dicha competencia (Sanches, 2013).

Para el desarrollo del programa se sigue una metodología basada en la investigación acción (IA) ya que, según manifiesta Alberich (2002), pretendemos obtener datos fiables para establecer mejoras en los mismos grupos de sujetos que son investigados. Desde la misma perspectiva se manifiesta Cruz Murillo (2015) cuando establece que este método involucra a los actores de situaciones educativas con el propósito de mejorarlas. Para ello, el investigador asumirá un rol de experto utilizando aquellas técnicas y/o estrategias que mejor se adapten al contexto.

Es por ello por lo que el PM se estructura con las cuatro fases establecidas por Kemmis y Mc'Taggart (1988) para el paradigma de IA:

La observación, considerando lo legislado en el punto 1 del artículo 12 del Decreto 97/2015, de 3 de marzo, por el que se establece la ordenación y el currículo de la EP en la Comunidad Autónoma de Andalucía, se aplican estrategias de evaluación continua:

a) La planificación, con el trabajo coordinado de diferentes docentes.

b) La acción, aplicando el programa de intervención que se considera más ajustado a las necesidades detectadas.

c) La reflexión, a partir de los datos obtenidos con las pruebas estadísticas y que nos aportan información para la toma de decisiones en el proceso de evaluación formativa que se explicita en el punto 2 del artículo 12 del mismo Decreto.

\section{Método}

\section{Diseño}

Se realiza un estudio cuantitativo con tres medidas (septiembre, diciembre y marzo) realizadas a un grupo experimental $(\mathrm{n}=14)$. La variable independiente que se establece es el programa para el aumento de la VL que realiza el alumnado seleccionado, mientras que las variables dependientes (VVDD) son el número de palabras leídas en un minuto, el tiempo total empleado para leer las tres tarjetas y el número de errores cometidos en la lectura de las tres tarjetas.

\section{Análisis estadístico}

El análisis estadístico se desarrolla con un 95\% de intervalo de confianza $(\mathrm{p}<0,05)$, utilizando para la gestión de los datos la aplicación informática IBM SPSS 23.0 Statistics.

Se realiza un estudio descriptivo de las tres VVDD, para cada uno de los meses en los que se realizan las medidas (tablas 2,3 y 4 ).

Se aplican pruebas de normalidad Kolmogorov-Smirnov (K-S) y homocedasticidad (Levene) en cada una de las medidas (tablas 5 y 6 ).

Se realiza un análisis de Modelo Lineal General (MLG) con medidas repetidas, aplicándose la eta al cuadrado parcial (tabla 7).

Se utiliza un ANOVA con un estudio post hoc con DNS (gráficos 1, 2 y 3).

\section{Muestra}

La muestra objeto de la intervención, está formada por 14 individuos seleccionados entre el alumnado de EP que puntuaba por debajo del límite inferior del intervalo de VL correspondiente a su edad, según la prueba de lectura de palabras de Cuetos, Rodríguez, Ruano y Arribas (2014).

Con la aplicación de dicho instrumento, se observa que de los 14 participantes que no superan el límite inferior de intervalo de VL, 10 no obtienen unos resultados adecuados en la prueba de seguimiento ocular de King, Gissane, Hume y Flaws (2015), conformándose la muestra final. 
Tabla 1

Muestra

\begin{tabular}{|c|c|c|}
\hline & Niños & Niñas \\
\hline $\mathrm{N}$ & 5 & 5 \\
\hline Edad (M) & 8,8 & 8,4 \\
\hline Edad (SD) & 0,45 & 0,55 \\
\hline Tercer Nivel & 1 & 1 \\
\hline Cuarto Nivel & 2 & 4 \\
\hline Quinto Nivel & 2 & 0 \\
\hline
\end{tabular}

Nota: N: Muestra, M: Media, SD: Desviación Típica.

\section{Procedimiento}

\section{Instrumento}

En este trabajo se utiliza el test King-Devick (K-D), un instrumento que fue desarrollado como una herramienta para evaluar la relación entre el déficit de las funciones oculomotoras y las dificultades de aprendizaje, es una prueba rápida y sencilla en su corrección que cualquier docente puede utilizar (Lieberman, Cohen y Rubin, 1983). Para ello, se aplica una tarjeta de demostración y posteriormente tres cartas más con cuarenta cifras situadas a diferentes distancias en cada una de ellas, evitando así la interpretación que acarrea la lectura de palabras (King et al., 2015).

\section{Programa de Intervención}

El programa de intervención se ha realizado en dos periodos de tres meses cada uno (septiembre-diciembre y diciembre-marzo) y, en cada uno de los trimestres, se han implementado 24 sesiones de 10 minutos, haciéndose en dos días a la semana. Los alumnos/as salían, por pares, de su grupo clase dirigiéndose a un aula en la que trabajaban dos actividades en cada jornada.

Las actividades aplicadas están diseñadas por García Castellón (2013) y son las siguientes: Actividad 1

\begin{tabular}{ll}
\hline Título & Seguir la linterna \\
Objetivo & Ampliar los movimientos oculares en todas las posiciones de mirada \\
Nivel & A partir de los 3 años \\
Tiempo & 2 minutos \\
Materiales & Una linterna pequeña \\
& $\begin{array}{l}\text { Al sujeto se le instruirá para que siga con los ojos la luz de la linterna, y se le } \\
\text { pedirá que no mueva ninguna otra parte del cuerpo. El ojo deberá moverse tan } \\
\text { Descripción } \\
\end{array}$ \\
& $\begin{array}{l}\text { suavemente como lo haga la linterna. Se sitúa a } 40 \mathrm{~cm} \text { de la cara y se desplaza en } \\
\text { horizontal y vertical. }\end{array}$ \\
\hline
\end{tabular}

Actividad 2

Título El reloj

Objetivo Mejorar los movimientos oculares y la flexibilidad

Nivel A partir de los 3 años

Tiempo 2 minutos

Materiales Un reloj con números en círculo

Pedir que se situé de pie o sentado a 1 metro de distancia, con la espalda y la

Descripción cabeza rectos y con los pies juntos. Indicarle que tiene que mirar el número que le vamos a decir y mantener la fijación hasta que le digamos otro. 
Actividad 3

Título Laberintos

Objetivo Mejorar la motricidad fina y la coordinación ojo-mano

Nivel A partir de los 4 años

Tiempo 2-3 minutos

Materiales Laberintos impresos, puntero láser

Seguir los laberintos con el puntero láser y en una segunda pasada con el dedo.

Descripción La espalda y la cabeza tienen que permanecer rectas facilitando que el recorrido sea seguido con movimientos oculares.

Actividad 4

Título Lectura global

Objetivo Realizar lecturas globales de palabras

Nivel A partir de 6 años

Tiempo 3-4 minutos

Materiales Tarjetas con palabras

Los participantes tendrán que leer una serie de palabras y deberán leerlas con

Descripción velocidad para que adquieran la lectura global. De modo que en cada fijación pueda ver la palabra y no tenga que ir silabeando. Las primeras palabras tienen un menor número de sílabas aumentándolas progresivamente.

Actividad 5

Título Movimientos con seguimientos

Objetivo Mejorar la motricidad, la fijación y la coordinación ojo-mano.

Nivel A partir de los 3 años

Tiempo 1-2 minutos

Materiales Un lápiz

En posición sentada o de pie, dar al niño/a un lápiz e indicarle que fije la mirada

Descripción en la punta. Ha de moverlo describiendo grandes círculos a derecha e izquierda y seguirlo con los ojos sin mover la cabeza.

Actividad 6

Título Fijación de lápices de colores

Objetivo Afianzar la fijación de los movimientos sacádicos

Nivel A partir de 5 años

Tiempo 1-2 minutos

Materiales Lápices de diferentes colores

Descripción Los participantes tendrán que mirar la punta de un lápiz de color o de otro, indistintamente siguiendo las indicaciones del profesor/a.

Actividad 7

Título Leer la primera y la última palabra de una línea

Objetivo Aumentar la distancia de fijación en los movimientos sacádicos

Nivel A partir de 5 años

Tiempo 3-4 minutos

Materiales Texto adecuado a la edad del participante

Descripción Los participantes tendrán que leer la primera y la última letra de una línea. 
Después la siguiente, hasta terminar el texto de manera que mueva sus ojos adecuadamente.

Actividad 8

Título Textos separados

Objetivo Leer textos separados en columnas

Nivel A partir de 6 años

Tiempo 3-4 minutos

Materiales Texto separado en dos columnas hacia la derecha e izquierda

Descripción Los participantes deben leer un texto que separado por dos columnas, cada una de ellas en direcciones opuestas favoreciendo el movimiento de los ojos.

Actividad 9

Título Búsqueda de palabras

Objetivo Buscar palabras

Nivel A partir de los 5 años

Tiempo 3-4 minutos

Materiales Texto adecuado al nivel del alumno/a con palabras repetidas en el mismo párrafo

Descripción Los participantes tendrán que buscar las palabras que se repiten en un texto.

Actividad 10

Título Movimientos verticales, horizontales y oblicuos

Objetivos Mejorar los movimientos en vertical, horizontal y oblicuo

Mantener la fijación

Nivel A partir de los 3años

Tiempo 1-2 minutos

Materiales Ninguno

En posición sentada o de pie dirigir la mirada hacia distintos puntos de manera

Descripción que se mueva la mirada de izquierda a derecha, de arriba abajo, parpadear, mantener la mirada, etc...

\section{Resultados}

Tras someter los datos a las pruebas estadísticas descritas con anterioridad se obtienen los siguientes resultados:

Tabla 2

Descriptivos septiembre

\begin{tabular}{lcccccc}
\cline { 2 - 6 } & $\mathrm{N}$ & $\mathrm{M}$ & $\mathrm{SD}$ & Asim. & Curt. & $\mathrm{R}$ \\
\hline $\begin{array}{l}\text { Número de palabras leídas por } \\
\text { minuto }\end{array}$ & 10 & 58,80 & 13,742 & 0,027 & $-1,031$ & 43 \\
$\begin{array}{l}\text { Tiempo total de lectura de las tres } \\
\text { tarjetas }\end{array}$ & 10 & 99,50 & 17,507 & 0,303 & $-0,767$ & 53 \\
$\begin{array}{l}\text { Número de errores totales en las tres } \\
\text { tarjetas }\end{array}$ & 10 & 11,20 & 6,828 & 1,841 & 4,100 & 24 \\
\hline
\end{tabular}

Nota: N: Muestra, M: Media, SD: Desviación Típica, Asim.: Asimetría, Curt.: Curtosis y R: Rango. 
Tabla 3

Descriptivos diciembre

\begin{tabular}{lcccccc}
\cline { 2 - 6 } & $\mathrm{N}$ & $\mathrm{M}$ & $\mathrm{SD}$ & Asim. & Curt. & $\mathrm{R}$ \\
\hline Número de palabras leídas por minuto & 10 & 84,20 & 26,939 & 1,058 & 0,748 & 86 \\
$\begin{array}{l}\text { Tiempo total de lectura de las tres tarjetas } \\
\begin{array}{l}\text { Número de errores totales en las tres } \\
\text { tarjetas }\end{array}\end{array}$ & 10 & 83,80 & 11,998 & 1,060 & 2,609 & 43 \\
\hline
\end{tabular}

Nota: N: Muestra, M: Media, SD: Desviación Típica, Asim.: Asimetría, Curt.: Curtosis y R: Rango.

Tabla 4

Descriptivos marzo

\begin{tabular}{lcccccc}
\cline { 2 - 6 } & $\mathrm{N}$ & $\mathrm{M}$ & $\mathrm{SD}$ & Asim. & Curt. & $\mathrm{R}$ \\
\hline $\begin{array}{l}\text { Número de palabras leídas por } \\
\text { minuto }\end{array}$ & 10 & 89 & 21,525 & $-0,503$ & $-0,857$ & 63 \\
$\begin{array}{l}\text { Tiempo total de lectura de las } \\
\text { tres tarjetas }\end{array}$ & 10 & 78,70 & 13,191 & 0,257 & $-0,950$ & 40 \\
$\begin{array}{l}\text { Número de errores totales en } \\
\text { las tres tarjetas }\end{array}$ & 10 & 1 & 1,155 & 2,165 & 5,973 & 4 \\
\hline Nota: N:
\end{tabular}

Nota: N: Muestra, M: Media, SD: Desviación Típica, Asim.: Asimetría, Curt.: Curtosis y R: Rango.

Tabla 5

Prueba de normalidad

\begin{tabular}{|c|c|c|c|c|c|c|}
\hline & \multirow{2}{*}{\multicolumn{2}{|c|}{ septiembre }} & \multirow{2}{*}{\multicolumn{2}{|c|}{ diciembre }} & \multirow{2}{*}{\multicolumn{2}{|c|}{ marzo }} \\
\hline & & & & & & \\
\hline & $\begin{array}{l}\mathrm{Z} \text { de } \\
\mathrm{K}-\mathrm{S}\end{array}$ & p. & $\begin{array}{l}\text { Z de } \\
\text { K-S }\end{array}$ & p. & $\begin{array}{l}\mathrm{Z} \text { de } \\
\mathrm{K}-\mathrm{S}\end{array}$ & p. \\
\hline $\begin{array}{l}\text { Número de palabras leídas por } \\
\text { minuto }\end{array}$ & 0,466 & 0,982 & 0,826 & 0,502 & 0,497 & 0,966 \\
\hline $\begin{array}{l}\text { Tiempo total de lectura de las } \\
\text { tres tarjetas }\end{array}$ & 0,354 & 1 & 0,641 & 0,806 & 0,605 & 0,857 \\
\hline $\begin{array}{l}\text { Número de errores totales en } \\
\text { las tres tarjetas }\end{array}$ & 0,801 & 0,542 & 0,771 & 0,592 & 1,265 & 0,082 \\
\hline
\end{tabular}

Nota: Z de K-S: Estadístico de Kolmogorov-Smirnov, p: Significatividad bilateral.

Los resultados mostrados en la Tabla 5 demuestran que las distribuciones de las tres VVDD presentan características de normalidad. Por lo que se estima oportuno realizar un estudio de igualdad de varianzas. 
Tabla 6

Homocedasticidad

\begin{tabular}{|c|c|c|c|c|}
\hline & $\mathrm{F}$ & gl1 & $\mathrm{gl} 2$ & p. \\
\hline $\begin{array}{l}\text { Número de palabras leídas por } \\
\text { Minuto }\end{array}$ & 2,086 & 2 & 27 & 0,144 \\
\hline $\begin{array}{l}\text { Tiempo total de lectura de las } \\
\text { tres tarjetas }\end{array}$ & 1,312 & 2 & 27 & 0,286 \\
\hline $\begin{array}{l}\text { Número de errores totales en } \\
\text { las tres tarjetas }\end{array}$ & 5,329 & 2 & 27 & 0,011 \\
\hline
\end{tabular}

Nota: F: Estadístico de Leven, gl1: grados de libertad, p: Significatividad bilateral.

Se observa igualdad de varianzas en las dos primeras variables, mientras que no existe en la variable Número de errores totales en las tres tarjetas.

Al comprobar las características de normalidad y homocedasticidad que presentan las distribuciones de las VVDD y, al existir medidas en el mismo grupo de sujetos en tres momentos diferenciados en el tiempo se realiza un análisis de MLG con medidas repetidas, aplicándose la eta al cuadrado parcial $(\eta 2 \mathrm{p})$ para comprobar el tamaño del efecto (TE) que el programa de intervención produce. Entendiéndose la $\eta 2$ p como la proporción de cada VD que es explicada por la VI (Cárdenas y Arancibia, 2014).

A pesar del tamaño muestral y de no existir igualdad de varianzas en la variable número de errores, se considera adecuado la utilización de pruebas paramétricas porque el sesgo no es exagerado y la potencia del ANOVA permite superarlo (Schmider, Ziegler, Danay, Beyer y Bühner, 2010).

Tabla 7

Prueba de los efectos intra-sujetos

\begin{tabular}{|c|c|c|c|c|c|}
\hline & $\mathrm{F}$ & gl1 & $\mathrm{g} 12$ & p. & $\eta_{p}^{2}$ \\
\hline $\begin{array}{l}\text { Número de palabras leídas por } \\
\text { Minuto }\end{array}$ & 28,66 & 2 & 18 & 0,001 & 0,761 \\
\hline $\begin{array}{l}\text { Tiempo total de lectura de las } \\
\text { tres tarjetas }\end{array}$ & 14,76 & 2 & 18 & 0,001 & 0,621 \\
\hline $\begin{array}{l}\text { Número de errores totales en } \\
\text { las tres tarjetas }\end{array}$ & 23,75 & 2 & 18 & 0,001 & 0,725 \\
\hline
\end{tabular}

Nota: F: Estadístico del ANOVA, g11: grados de libertad, p: Significatividad bilateral, $\eta 2 p$ : Eta al cuadrado parcial.

Tras la realización de la prueba de los efectos intra-sujetos que se realiza a las tres VVDD se aprecia que el tamaño que tiene la $\eta 2 p$ demuestra la gran incidencia que tiene la VI sobre las VVDD. 


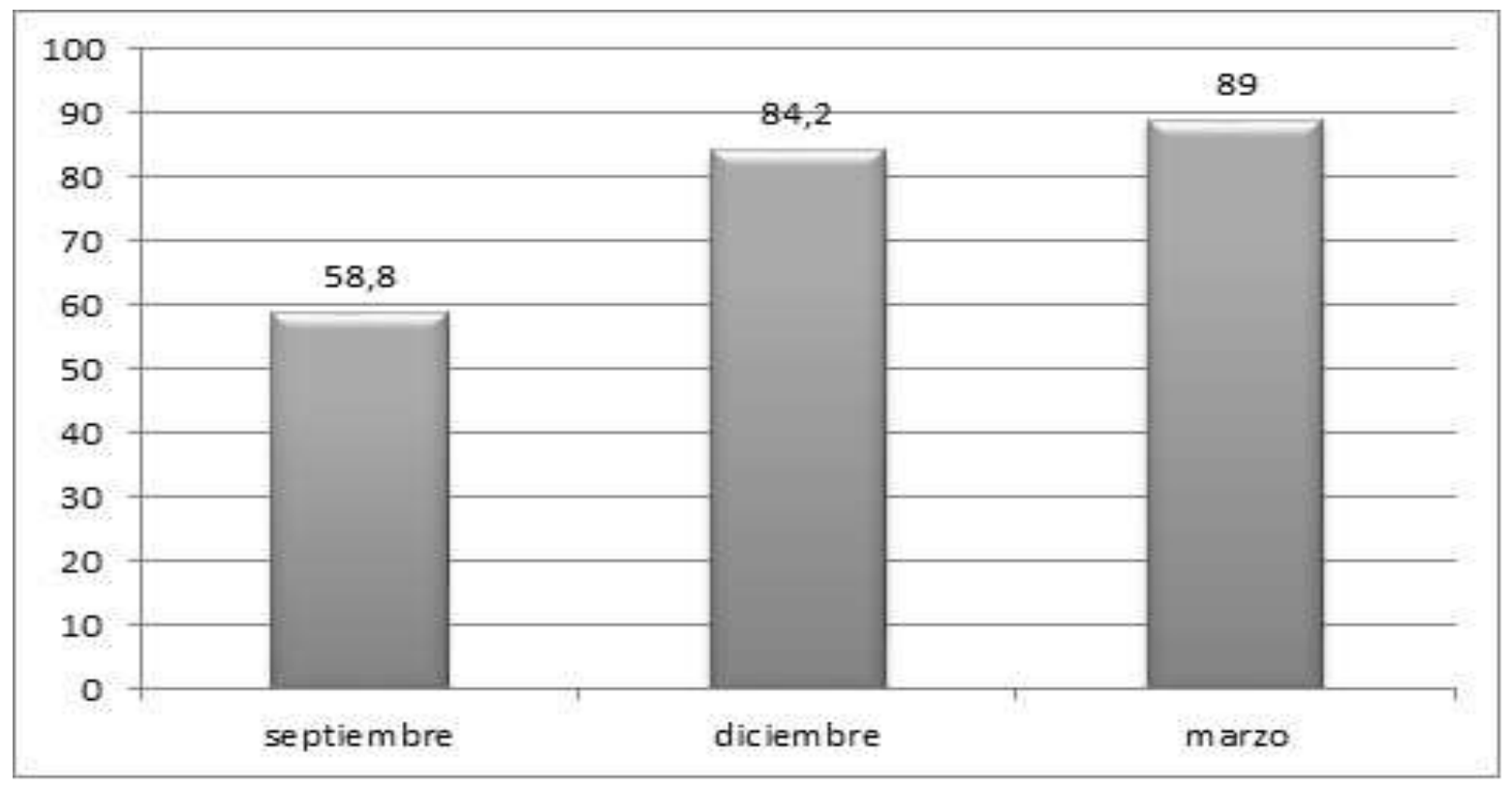

Gráfica 1. Comparación por pares con DNS entre las tres medidas en la variable Número de palabras leídas por minuto.

Se puede observar que entre el mes de septiembre y el de diciembre hay una diferencia de medias (DM) de 25,4; $\mathrm{p}<0,01$, entre la de septiembre y la de marzo existe una DM de 30,2; $\mathrm{p}<0,001$ $\mathrm{y}$, por último, entre el mes de diciembre y el de marzo se obtiene una DM de 4.8; $\mathrm{p}>0,05$.

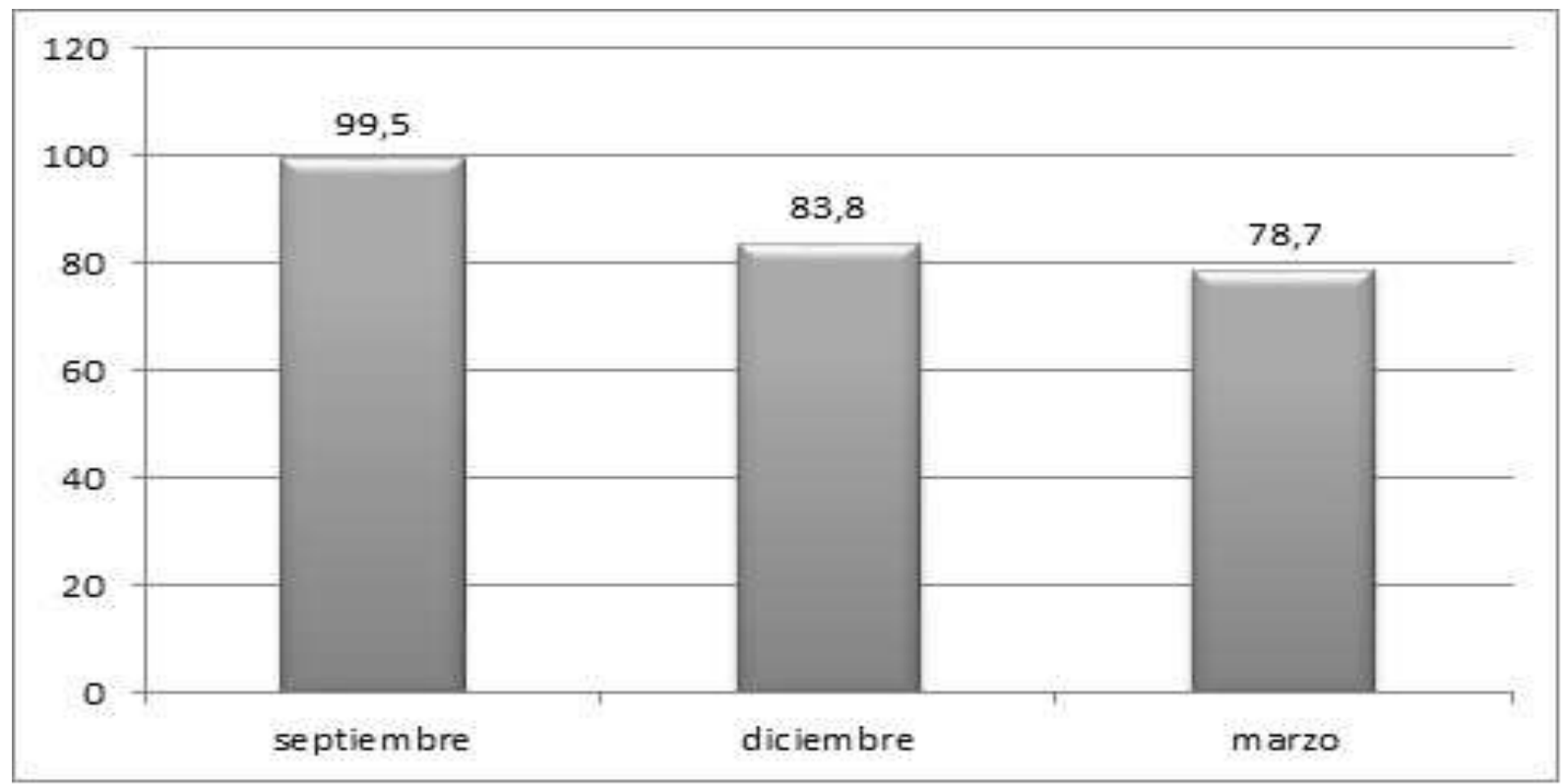

Gráfica 2. Comparación por pares con DNS entre las tres medidas en la variable Tiempo total de lectura de las tres tarjetas. 
En la comparación por pares con DNS existe una DM de 15; $\mathrm{p}<0,01$ entre los meses de septiembre y diciembre, una DM de 20,8; $\mathrm{p}<0,01$ entre la medida de septiembre y marzo y una DM de 5,1; $>>0,05$ entre las de diciembre y marzo.

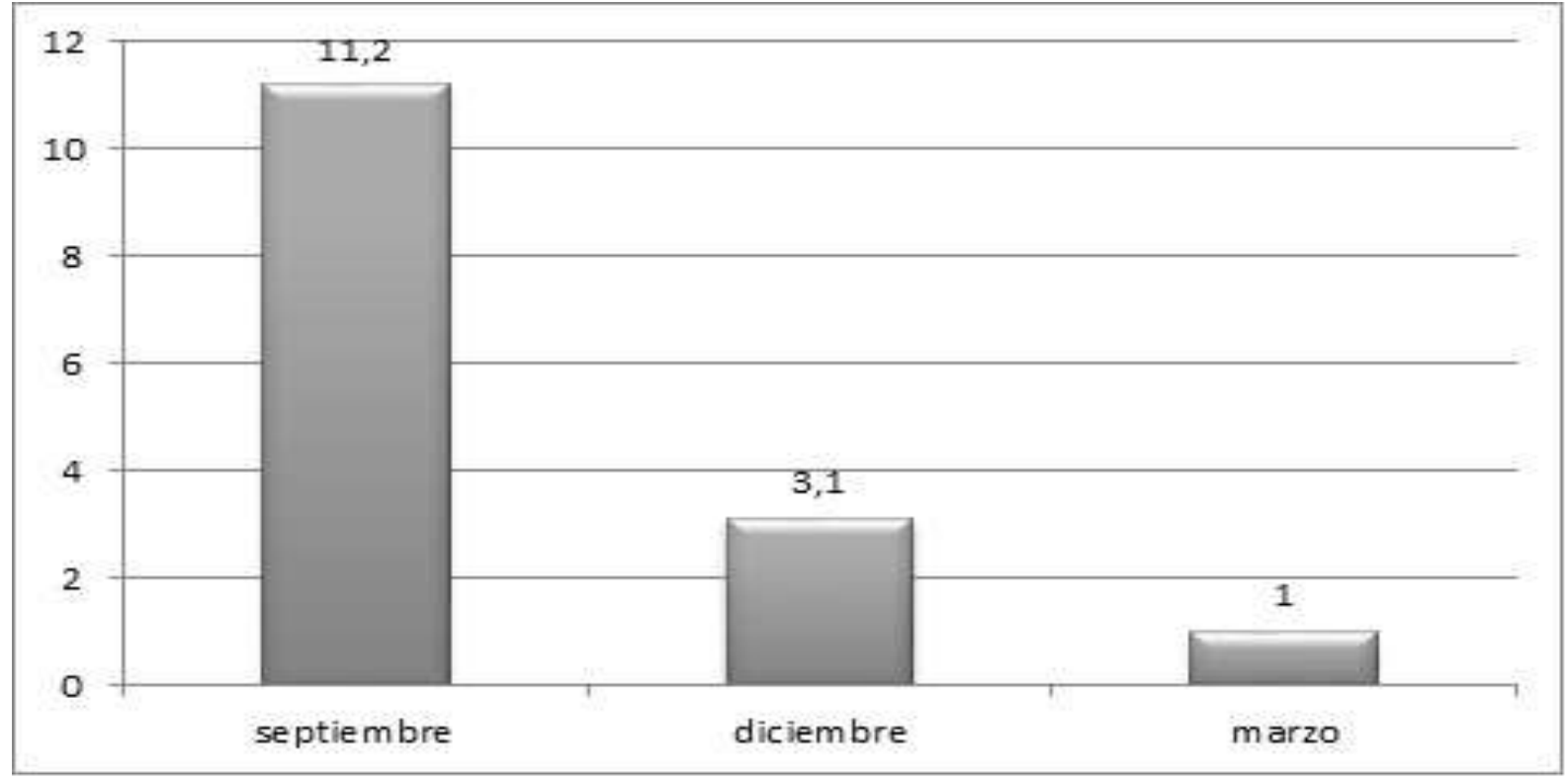

Gráfica 3. Comparación por pares con DNS entre las tres medidas en la variable Número de errores totales en las tres tarjetas.

$\mathrm{Al}$ realizar la comparación por parejas con DNS se accede a una DM 8,1; $\mathrm{p}<0,01$ entre los meses de septiembre y diciembre, una DM de 10,2; $\mathrm{p}<0,001$ entre las medidas de septiembre y marzo y una DM de 2,1; $<<0,01$ entre diciembre y marzo.

\section{Discusión}

Las conclusiones de diferentes programas de entrenamiento de saltos sacádicos han demostrado que la mejora y la reducción de los movimientos oculares se relacionan directamente con la velocidad y CL (Rayner, Slattery y Bélanger, 2010), del mismo modo se ha comprobado que al aumentar la velocidad se reducen los movimientos oculares (Miyata, Minagawa, Watanabe, Sasaki y Ueda 2012; Solan, Shelley, Ficarra, Silverman y Larson, 2003), quedando demostrada la relación entre el tiempo de lectura y la eficacia en ella (Megino, Martín-Lobo y Vergara, 2016).

Los resultados obtenidos en este trabajo, afianzan los propuestos por Kujala, Karma, Ceponiene, Belitz, Turkkila, Tervaniemi y Näätänen (2001); Kenji, Tsuyoshi y Shinya (2011); Kim (2016); Kuperman et al. (2016) ya que se ha conseguido aumentar la VL, reducir el tiempo de lectura de las tres tarjetas y disminuir el número de errores.

Se observa que para la mejora de las dos primeras VVDD, solo es necesario un trimestre, ya que las diferencias que se obtienen en el segundo no son estadísticamente significativas. Además, al estudiar el resultado que se ha obtenido en la $\eta 2 p$, se puede afirmar que el TE que ha producido la intervención, ha sido grande (Cárdenas y Arancibia, 2014).

Los programas de entrenamiento de los movimientos oculares también han producido resultados estadísticamente significativos cuando se asocian a variables ambientales, tal es el caso del 
trabajo desarrollado por Navarro, Vallejo y Sicilia (1998), que implementaron en dos aulas de sexto nivel de primaria, modificando el contexto en una de ellas. La intervención se realizó a un grupo de sujetos de ambas unidades, obteniendo mejoras significativas en los discentes de las dos unidades, aunque más acentuadas en aquellos que pertenecían al aula modificada.

Tras el análisis de las variables estudiadas, se está en disposición de ofrecer un PM que incide directamente en la precisión lectora del alumnado. Este trabajo promueve la mejora educativa partiendo de un paradigma cuantitativo que persigue encontrar soluciones a los problemas que aparecen en los procesos de enseñanza-aprendizaje (Castellano y Pantoja, 2017), compartiendo lo explicitado por Carr (2002) cuando defiende que el estudio de la relación entre la teoría pedagógica y la práctica educativa, ha recibido gran parte de los trabajos realizados por la comunidad científica. Pero, a pesar de estos esfuerzos, la abstracción de los contenidos teóricos permanecen alejados del día a día de las aulas, por lo que se asume lo establecido por Burkhardt y Schoenfeld (2003) cuando manifiestan que la investigación educativa debe estar relacionada con las necesidades prácticas de los sistemas educativos.

Por lo tanto, se puede afirmar que esta investigación se realiza para dar cumplimiento a la obligación que tienen los docentes de desarrollar todas aquellas intervenciones necesarias para una detección temprana de las dificultades lectoras de sus alumnos/as (Powers, Grisham y Riles, 2008).

\section{Conclusión}

Dando respuesta a los objetivos planteados en la introducción, y según los datos que nos aporta el análisis estadístico, se concluye que la aplicación de este programa de entrenamiento para la mejora de los movimientos oculares en la etapa de EP, consigue una mejora estadísticamente significativa en todas las VVDD, favoreciendo los procesos de automatización y fluidez lectora, imprescindibles para la comprensión de textos (Fumagalli, Barreyro y Jaichenco, 2017).

Aunque el presente trabajo tiene las limitaciones que se derivan del tamaño muestral, los resultados obtenidos nos disponen a defenderlo como un método válido, funcional y extrapolable al resto de centros educativos, dando cumplimiento a lo establecido en el punto 2 del artículo 19 de la Ley Orgánica 2/2006, de 3 de mayo, de Educación, en relación a la necesidad que tienen los docentes de organizar su práctica para garantizar un adecuado nivel de desempeño de la competencia lingüística.

\section{Referencias}

Alberich, T. (2002). Perspectivas de la Investigación Social. En J. Martí, M. Montañés, y T. Rodríguez-Villasante (Coords), La Investigación Social Participativa (pp. 65-78). Madrid: El Viejo Topo.

Alonso-Tapia, J. (2005). Claves para la enseñanza de la comprensión lectora. Revista de Educación, núm. extraordinario 2005, 63-93.

Bernardo, J. (2004). Estrategias de aprendizaje: para aprender más y mejor. Madrid: RIALP.

Burkhardt, H. \& Schoenfeld, A. (2003). Improving educational research: Toward a more useful, more influential and better-funded enterprise. Educational Researcher, 32(9), 3-14.

Cárdenas-Castro, M. y Arancibia, H. (2014). Potencia estadística y cálculo del tamaño del efecto en g*power: complementos a las pruebas de significación estadística y su aplicación en psicología. Salud \& Sociedad, 5(2), 210-224. Disponible en: http//dx.doi.org/10.22199/s07187475.2014.0002.00006

Carr, W. (2002). Una teoría para la educación. Hacia una investigación educativa crítica. Madrid: Ediciones Morata, S. L. 
Castellano-Luque, E. A. y Pantoja-Vallejo, A. (2017). Eficacia de un programa de intervención basado en el uso de las TIC en la tutoría. Revista de Investigación Educativa, 35(1), 215-233. Disponible en: http://doi.org/10.6018/rie.35.1.248831

Cortés de las Heras, J. (2002). Estudio de perfiles evolutivos en la lectura validación y revisión del test individual de diagnóstico de errores en lectura (TIDEL) (Tesis Doctoral). Universitat de València, Valencia. Disponible en: http://www.tesisenred.net/bitstream/handle/10803/10089/TESIS.PDF?sequence=1

Cuetos, F., Rodríguez, B., Ruano, E., y Arribas, D. (2014). PROLEC-R. Batería de evaluación de los procesos lectores, Revisada ( $5^{a}$ edición). Madrid: TEA Ediciones.

Cruz-Murillo, J. A. (2015). Investigación Acción en la Educación. Dialéctica: Revista de Investigación Educativa, 1, 179-189.

Decreto 97/2015, de 3 de marzo, por el que se establece la ordenación y el currículo de la Educación primaria en la Comunidad Autónoma de Andalucía. BOJA, núm. 50 de 13 de marzo de 2015. Recuperada de https://www.juntadeandalucia.es/boja/2015/50/BOJA15-050-00012-461701_00065845.pdf

Fumagalli, J. C., Barreyro, J. P. y Jaichenco, V. V. (2017). Fluidez lectora en niños: cuáles son las habilidades subyacentes. Ocnos, 16(1), 50-61. Disponible en: https://doi.org/10.18239/ocnos_2017.16.1.1332

Galindo, J., y Martínez I. (2014). Fortaleciendo la Motivación Mediante Estrategias de Comprensión Lectora en Estudiantes de Educación Primaria. Innovare, Revista de ciencia y tecnología, 3(2), 11-25. Disponible en: http//dx.doi.org/10.5377/innovare.v3i2.2305

García-Castellón, M. C. (2013). Funcionalidad visual y eficacia en los procesos lectores. Logroño: Universidad Internacional de La Rioja, S.A.

Gómez, L. F. (2008). El desarrollo de la competencia lectora en los primeros grados de primaria. Revista Latinoamericana de Estudios Educativos, 38(3 y 4), 95-126.

Guzmán Torres, L. T., Fajardo, M. E., y Duque, C. P. (2015). Comprensión y producción textual narrativa en estudiantes de educación primaria. Revista Colombiana de Psicología, 24(1), 61-83. Disponible en: http://doi.org/10.15446/rcp.v24n1.42314

Kemmis, S. y Mc Taggart, R. (1988). Como planificar la Investigación-Acción. Barcelona: Laertes.

Kenji, Y., Tsuyoshi, T. \& Shinya, S. (2011). Improvement of Reading Speed and Eye Movements. IPerception, 2(4), 263-264. Disponible en: http//dox.doi.org/10.1068/ic263

Kim, J. S. (2016). The Effects of Chunking Speed Reading on Improving Reading Speed and Comprehension. Contemporary Research Trend of Education, 5, 69-71. Disponible en: http://doi.org/10.21742/asehl.2016.5.17

King, D., Gissane, C., Hume, P. A. \& Flaws, M. (2015). The King-Devick test was useful in management of concussion in amateur rugby union and rugby league in New Zealand. Journal of the Neurological Sciences, 351(1-2), 58-64. Disponible en: http//dx.doi.org/10.1016/j.jns.2015.02.035

Kujala, T., Karma, K., Ceponiene, R., Belitz, S., Turkkila, P., Tervaniemi, M. \&, Näätänen, R. (2001). Plastic neural changes and reading improvement caused by audiovisual training in readingimpaired children. Proceedings of National Academy of Sciences of the United States of America, 18(98), 10509-10514. Disponible en: http://doi.org/10.1073/pnas.181589198

Kulp, M. T. \& Schmith, P. P. (1997). The relation of clinical saccadic eye movement testing to reading in hindersgartens and first graders. Optometry Vision Sciencie, 74, 37-42. Disponible en: http//dx.doi.org/10.1097/00006324-199701000-00018

Kuperman, V., Van Dyke, J. \& Henry, R. (2016). Eye-Movement Control in RAN and Reading. Journal Scientific Studies of Reading, 20, 173-188. Disponible en: http//doi.org/10.1080/10888438.2015.1128435 
La Cámara, J. M. (2016). Relación entre eficacia de los saltos sacádicos y proceso lector en estudiantes de currículo específico en Educación Secundaria. Alcoy: Editorial Área de Innovación y Desarrollo. Disponible en: http//dx.doi.org/10.17993/dideinnedu.2016.10

Lieberman, S., Cohen, A.H. \& Rubin, J. (1983). NYSOA K-D test. Journal Optometry Association, 54(7), 631-637.

León, J. A. (2004). ¿Por qué las personas no comprenden lo que leen? Psicología Educativa, 10(2), 101-116.

Ley Orgánica 2/2006, de 3 de mayo, de Educación, Boletín Oficial del Estado, núm. 106, de 4 de mayo de 2006. Recuperada de https://www.boe.es/boe/dias/2006/05/04/pdfs/A1715817207.pdf

Megino-Elvira, L., Martínn-Lobo, P. \& Vergara-Moragues, E. (2016). Influence of eye movements, auditory perception, and phonemic awareness in the reading process. The journal of educational research, 109(6), 567-573. Disponible en: https://doi.org/10.1080/00220671.2014.994197

Melero, N. (2011). El paradigma crítico y los aportes de la investigación acción participativa en la transformación de la realidad social: un análisis desde las ciencias sociales. Cuestiones Pedagógicas, 21, 339-355.

Miyata, H., Minagawa, Y., Watanabe, S., Sasaki, T. \& Ueda, K. (2012). Reading speed, comprehension and eye movements while reading Japanese Novels: Evidence from untrained readers and cases of speed-reading trainees. PloS One, 7(5). e36091. Disponible en: https://doi.org/10.1371/journal.pone.0036091

National Reading Panel, National Institute of Child Health \& Human Development. (2000). Report of the national reading panel: Teaching children to read: An evidence-based assessment of the scientific research literature on reading and its implications for reading instruction: Reports of the subgroups. National Institute of Child Health and Human Development, National Institutes of Health. Disponible en:

https://www.nichd.nih.gov/sites/default/files/publications/pubs/nrp/Documents/report.pdf \#search=Report $\% 20$ of $\% 20$ the $^{2}$ 20 national\%20reading\%20panel:\%20Teaching\%20chi

Navarro, M. J., Vallejo, I. y Sicilia, S. (1998). Efecto del entrenamiento visual y la intervención ergonómica en el rendimiento de la lectura comprensiva: informe preliminar. Gaceta Óptica, $320,10-14$.

Palomo-Álvarez, C. (2010). Habilidades visuales en niños de educación primaria con problemas de lectura e influencia de un filtro amarillo en la visión y la lectura (Tesis Doctoral). Universidad Complutense de Madrid, Madrid.

Pascual, G., Goikoextea, E., Corral, S., Ferrero, M. y Pereda, V. (2014). La Enseñanza Recíproca en las Aulas: Efectos Sobre la Comprensión Lectora en Estudiantes de Primaria. Psykhe, 23(1), 112. Disponible en: http//dx. doi.org/10.7764/psykhe.23.1.505

Paulidis, G. T. (1981). Sequencing, eye movements and the early objetive diagnosis of dyslexia. New York: John Wiley \& Son.

Pinnell, G. S., Pikulski, J. J., Wixson, K., Campbell, J. R., Gough, P. B. \& Beatty, A. (1995). Listening to children read aloud. Washington, DC: U.S. Department of education, national center for education statistics.

Powers, M., Grisham, D. \& Riles, P. (2008). Saccadic tracking skills of poor readers in high school. Optometry, 79, 228-234. Disponible en: http://dx.doi.org/10.1016/j.optm.2007.07.014

Rayner, K., Slattery, T. J., \& Bélanger, N. N. (2010). Eye movements, the perceptual span, and reading speed. Psychonomic Bulletin \& Review, 17(6), 834-839. Disponible en: https://doi.org/10.3758/pbr.17.6.834

Sanches-Chévez, L. E. (2013). La comprensión lectora: hacia una aproximación sociocultural. Diálogos, 12, 7-16. Disponible en: http//dx.doi.org/10.5377/dialogos.v0i12.2191 
Scheiman, M., \& Wick, B. (1994). Clinical management of binocular visión: heterophoric, acommodative, and eye movement disorders. Philadelphia: JB Lippincott.

Schmider, E., Ziegler, M., Danay, E., Beyer, L. \& Bühner, M. (2010). Is it really robust? Reinvestigating the robustness of ANOVA against violations of the normal distribution assumption. Methodology, 6(4), 147-151. Disponible en: http//dx.doi.org/10.1027/1614$2241 / \mathrm{a} 000016$

Solan, A., Shelley-Tremblay, J., Ficarra, A., Silverman, M. \& Larson, S. (2003). Effect of attention therapy on reading comprehension. Learn Disabilities, 36(6), 556-563. Disponible en: https://doi.org/10.1177/00222194030360060601

\section{Información sobre los autores}

Autor: Ramón Cruz del Moral

Institución: Consejería de Educación de la Junta de Andalucía

Email: ramon.cruz.edu@juntadeandalucia.es

Autor: Sandra María Arjona Luque

Institución: Consejería de Educación de la Junta de Andalucía Email: - 


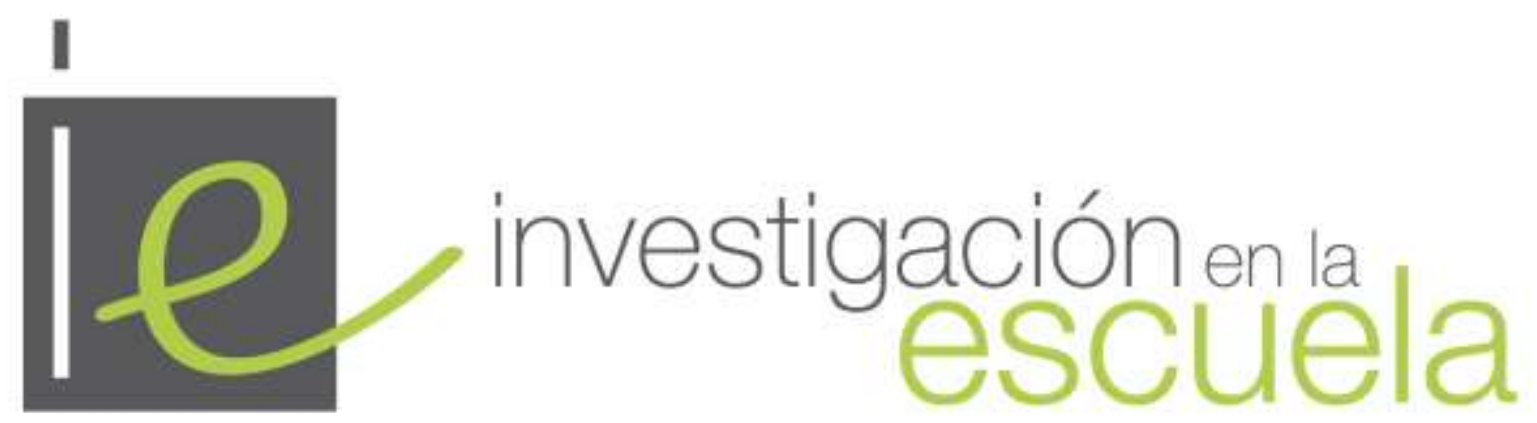

Revista académica evaluada por pares y de acceso abierto

Número 96

31 de diciembre de 2018

ISSN 2443-9991

\section{(c)}

SOMREFIIGHISRESERVEDLOS/as lectores/as pueden copiar, mostrar, y distribuir este artículo, siempre y cuando se de crédito y atribución al autor/es y a Investigación en la Escuela, se distribuya con propósitos no-comerciales, no se altere o transforme el trabajo original. Más detalles de la licencia de CreativeCommons se encuentran en http://creativecommons.org/licenses/by-nc-sa/3.0 Cualquier otro uso debe ser aprobado en conjunto por el autor/es, o Investigación en la Escuela.

ำ investigacion-en-la-escuela

Contribuya con comentarios y sugerencias en la web de la revista. Por errores y sugerencias contacte a secretaria@investigacionenlaescuela.es 


\section{Investigación en la escuela}

Consejo de dirección: Ana Rivero García (Universidad de Sevilla), Nicolás de Alba Fernández (Universidad de Sevilla), Pedro Cañal de León (Universidad de Sevilla), Francisco F. García Pérez (Universidad de Sevilla), Gabriel Travé González (Universidad de Huelva), Francisco F. Pozuelos Estrada (Universidad de Huelva)

Dirección: Ana Rivero García y Nicolás de Alba Fernández

Secretaría de edición: Elisa Navarro Medina

\section{Consejo editorial}

José Félix Angulo Rasco. Universidad de Cádiz Rosa Ma Ávila Ruiz. Universidad de Sevilla Pilar AzcárateGoded. Universidad de Cádiz Juan Bautista Martínez Rodríguez. Universidad de Granada

Nieves Blanco García. Universidad de Málaga Fernando Barragán Medero. Universidad de La Laguna José Carrillo Yáñez. Universidad de Huelva José Contreras Domingo. Universidad de Barcelona. Luis C. Contreras González. Universidad de Huelva Ana $\mathbf{M}^{\mathbf{a}}$ Criado García-Legaz. Universidad de Sevilla Rosario Cubero Pérez. Universidad de Sevilla José Ma Cuenca López. Universidad de Huelva Jesús Estepa Giménez. Universidad de Huelva Rafael Feito Alonso. Universidad Complutense (Madrid)

Francisco José García Gallardo. Universidad de Huelva

Soledad García Gómez. Universidad de Sevilla J. Eduardo García Díaz. Universidad de Sevilla
Fernando Hernández Hernández. Universidad de Barcelona

Salvador Llinares Ciscar. Universidad de Alicante Alfonso Luque Lozano. Universidad de Sevilla Rosa Martín del Pozo. Universidad Complutense (Madrid)

José Martín Toscano. IES Fernando Herrera (Sevilla) Jaume Martínez Bonafé. Universidad de Valencia F. Javier Merchán Iglesias. Universidad de Sevilla Emilia Moreno Sánchez. Universidad de Huelva. Rosario Ortega Ruiz. Universidad de Córdoba Antonio de Pro Bueno. Universidad de Murcia Fco. de Paula Rodríguez Miranda. Universidad de Huelva

Pedro Sáenz-López Buñuel. Universidad de Huelva Antoni Santisteban Fernández. Universidad Autónoma (Barcelona)

Emilio Solís Ramírez. Catedrático de IES. $\mathbf{M}^{\mathbf{a}}$ Victoria Sánchez García. Universidad de Sevilla. Magdalena Suárez Ortega. Universidad de Sevilla

\section{Consejo asesor}

Manuel Área Moreira. Universidad de La Laguna Jaume Carbonell. Director Cuadernos de Pedagogía. Barcelona César Coll. Universidad de Barcelona

Christopher Day. Universidad de Nothingham. U.K.

Juan Delval. Universidad Nacional de Educación a Distancia

John Elliott. Universidad de East Anglia. Norwich. U.K.

José Gimeno Sacristán. Universidad de Valencia

André Giordan. Universidad de Paris VII y Ginebra

Francisco Imbernón. Universidad de Barcelona Ángel Pérez Gómez. Universidad de Málaga

Rafael Porlán Ariza. Universidad de Sevilla

Francesco Tonucci. Instituto de Pedagogía del C.N.R. Roma

Jurjo Torres Santomé. Universidad de A Coruña 
